\title{
The concentration of insulin-like growth factor-1 in pregnancies complicated by pregnancy-induced hypertension and/or intrauterine hypotrophy
}

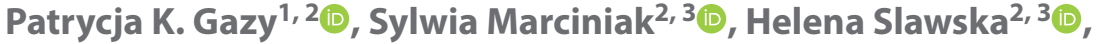 \\ Anita Olejek ${ }^{3}$, Bogdan Mazur ${ }^{1}$ (i) \\ ${ }^{1}$ Chair and Department of Microbiology and Immunology in Zabrze, Medical University of Silesia \\ ${ }^{2}$ Specialist Hospital No 2 in Bytom, Neonatal Unit No 5, Poland \\ ${ }^{3}$ Department of Gynecology, Obstetrics and Gynecologic Oncology in Bytom, School of Medicine with the Division of Dentistry in \\ Zabrze, Medical University of Silesia in Katowice, Bytom, Poland
}

\begin{abstract}
Objectives: The aim of the study was to compare Insulin-like Growth Factor-1 (IGF-1)concentration in pregnancies complicated by pregnancy-induced hypertension and/or intrauterine hypotrophy, and its correlation with maternal pressure and umbilical artery pulsatility and resistance indices.

Material and methods: 65 pairs pregnant-newborn were included to four groups: I - control, II - PIH, III - Hypotrophy, IV - PIH and Hypotrophy. In the study we analyzed cord blood IGF-1 concentration, newborns antropometry, umbilical artery pulsatility and resistance indices and maternal pressure before delivery.

Results: The concentration of IGF-1 was the lowest in IV group of hypotrophic newborns from pregnancies complicated by pregnancy-induced hypertension. In this group of patients there was strong negative correlation between IGF-1 concentration and maternal systolic and diastolic pressure.

Conclusions: There is a strong negative correlation between IGF-1 concentration and maternal systolic pressure in group of hypotrophic newborns from pregnancies complicated by pregnancy-induced hypertension.
\end{abstract}

Key words: intrauterine growth restriction; pregnancy-induced hypertension; insulin-like growth factor-1

Ginekologia Polska 2020; 91, 9: 544-548

\section{INTRODUCTION}

Pregnancy induced hypertension $(\mathrm{PIH})$ is one of the most serious complication of pregnancy significantly increasing the risk of stillbirth, prematurity, and perinatal death, both maternal and neonatal. Coexistence of pregnancy induced hypertension with intrauterine fetal hypotrophy increases these risks [1], but also has long-term negative impact on the further development of the children [2].

It is estimated that PIH occurs with varying intensity in $5-8 \%$ of all pregnancies [3], and their etiology is multifactorial and still unclear. It is believed that the basis for the development of this pathology are abnormal differentiation and invasion of extravillous trophoblast cells [4]. In healthy developing placentas, the extravillous trophoblast cells infiltrate spiral arteries, undergoing transformation from an epithelial to an endothelial phenotype. This leads to remodeling of their walls, conversion of muscles and elastic tissue localized in tunica media to fibrinous tissue. This process consists of two phases and enables transformation of these high resistance arteries into high capacitance, easily responsive to increased blood flow utero-placental vessels. The first stage includes the migration of trophoblast cells into the decidua. In the second stage, lasting from the $15^{\text {th }}$ to $20^{\text {th }}$ week of gestation, trophoblast cells infiltrate the spiral arteries. Abnormalities of the second phase was histologicaly observed in placentas from pregnancies complicated by $\mathrm{PIH}$, especially incomplete transformation of spiral arteries. This pathology can result in high resistance in placental vessels, significantly reducing placental blood flow. Impaired placental perfusion leads to fetal growth retardation, activation of inflammatory response cascade and damaging of endothelium $[5,6]$.

Intrauterine growth restriction occuring before the $32^{\text {nd }}$ week of gestation (early onset IUGR) is more often associ- 
ated with serious structural and functional abnormalities of the placenta, increased resistance in the placental vessels, haemodynamic changes with circulatory redistribution, and in the most severe cases centralization of fetal circulation. This form of IUGR is in 35\% associated with pregnancy-induced hypertension [7].

Umbilical arteries pulsatility and resistance indices are good parameters of fetal well-being status. Studies have also shown that screening doppler velocimetry between 20-24 weeks of gestation are good prognostic factors in assessing the risk of developing pregnancy-induced hypertension and intrauterine growth retardation [8].

\section{Objectives}

The aim of this study was a comparative analysis of IGF-1 concentrations in cord blood in three clinical situations: 1 . In pregnancies complicated by pregnancy-induced hypertension (PIH) and intrauterine fetal hypotrophy. 2. In pregnancies complicated by $\mathrm{PIH}$, with normal intrauterine growth of the fetus. 3. In pregnancies complicated by intrauterine fetal hypotrophy, without pregnancy-induced hypertension. An attempt was also made to assess the correlation between values of the pulsatility and resistance indices in umbilical arteries and maternal blood pressure on the concentration of insulin-like growth factor-1 in cord blood.

\section{MATERIAL AND METHODS}

In this study 65 pairs, pregnant-newborn, hospitalized in the Department of Gynecology and Obstetrics of the Specialist Hospital No. 2 in Bytom between 2015-2018 were assessed.

The pairs were included to one of four groups depending on the clinical situation:

- Control - eutrophic newborns from uncomplicated pregnancies;

- $\mathrm{PIH}$ - eutrophic newborns from pregnancies complicated by Pregnancy-Induced Hypertension;

- Hypo - Hypotrophic newborns from uncomplicated pregnancies;

- $\mathrm{PIH}+\mathrm{Hypo}$ - hypotrophic newborns from pregnancies complicated by Pregnancy-Induced Hypertension.

Exclusion criteria:

- prematurity < 32. weeks of gestation;

- multiple pregnancy;

- maternal diabetes;

- congenital diseases (including congenital defects, infections, persistent pulmonary hypertension);

- chronic maternal hypertension.

In all groups, the concentration of Insulin-like growth factor-1 (IGF-1) in the umbilical cord blood was tested by an immunoenzymatic method - ELISA with the use of Human IGF-1 Elisa Kit. The obtained results were analyzed in relation to mean values of systolic and diastolic blood pressure evaluated in the last day before pregnancy termination, pulsation index value and resistance index in umbilical arteries, based on routine pre-natal ultrasound examination with Color Doppler option. Neonatal anthropometric parameters (body length, birth weight, head circumference, chest circumference) were also analyzed.

Statistical analysis of the collected research material was based on the STATISTIKA statistical package, version 9.4. Distribution of quantitative variables was characterized by the mean and median values, standard deviation and standard error. The conformity assessment of the distribution of quantitative variables with normal distribution was performed using the Shapiro-Wilk test. Statistical significance of differences in distributions of quantitative variables between research groups was carried out using analysis of variance (ANOVA), with the assumption of homogeneity variations, whereas in the case where the variable distribution deviate from the normal, or in case of failure assumption of homogeneity of variance, Kruskal-Wallis and non-parametric U Mann-Whitney test was applied. Simple analysis was summarized multivariate regression analyzes. Statistical inference is based on the criterion of statistical significance of $a=0.05$.

\section{RESULTS}

Based on the analysis, it was observed that the concentration of insulin-like growth factor- 1 was the lowest in the group of hypotrophic newborns from pregnancies complicated by pregnancy-induced hypertension and was significantly lower compared to eutrophic newborns from normal pregnancies and pregnancies complicated by pregnancy-induced hypertension. In the groups with isolated hypotrophy or pregnancy-induced hypertension, IGF-1 concentrations were also lower than in healthy newborns, but without statistical significance (Fig. 1).

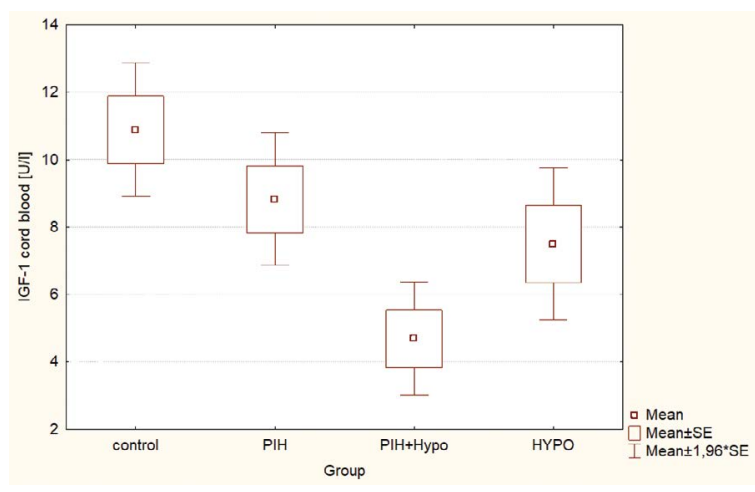

Figure 1. Comparative analysis of mean concentration of IGF-1 in cord blood in study groups with standard error and the $95 \%$ confidence interval 
Table 1. Patients characteristics - median values with the lowest and the highest values and standard deviations in parentheses

\begin{tabular}{|c|c|c|c|c|}
\hline & Control & PIH & Hypo & PIH + Hypo \\
\hline$N$ & 25 & 14 & 16 & 10 \\
\hline Maternal age & $30(6)$ & $34(5)$ & $34(6)$ & $33(4)$ \\
\hline Maternal BMI [kg/m^2] & $28.8(4.4)$ & $35.3(4.6)$ & $29.5(4.5)$ & $30.3(6.47)$ \\
\hline Maternal Weight [kg] & $78.5(12.9)$ & $95.2(14.7)$ & $77.4(13.2)$ & $86.7(16)$ \\
\hline $\mathrm{Hbd}$ & $37(34-41)$ & $36(32-41)$ & $37(34-40)$ & $34^{C T R, H}(32-41)$ \\
\hline B.W. [g] & $3190(2170-4480 ; 492.5)$ & $2870(1960-3800 ; 580)$ & $2257^{\text {CTR, P }}(1600-2900 ; 352)$ & $1550^{C T R, P}(840-2920 ; 562)$ \\
\hline Lenght $[\mathrm{cm}]$ & $53.1(47-60 ; 2.5)$ & $52.5(47-60 ; 4.0)$ & $49^{\text {CTR }}(43-54 ; 3)$ & $43.0^{C T R, P}(35-55 ; 5.2)$ \\
\hline Head circ. [cm] & $35(31-38 ; 2)$ & $34(30-37 ; 2)$ & $32.0^{C T R}(30-34 ; 1)$ & $29.0^{C T R, P}(24-32 ; 2.2)$ \\
\hline Chest circ.[cm] & $33(29-37 ; 2)$ & $31(27-34 ; 2)$ & $29.0^{C T R, P}(26-33 ; 2)$ & $25.0^{C T R, P}(20-32 ; 3.4)$ \\
\hline $\mathrm{Pl}$ in UA & $0.86(0.52-1.26 ; 0.17)$ & $0.86(0.55-1.18 ; 0.20)$ & $0.90(0.63-1.44 ; 0.24)$ & $1.1(0.81-1.9 ; 0.32)$ \\
\hline $\mathrm{Rl}$ in UA & $0.57(0.39-0.75 ; 0.08)$ & $0.56(0.40-0.68 ; 0.08)$ & $0.59(0.46-0.79 ; 0.09)$ & $\mathbf{0 . 7 0} \mathbf{C T R}^{\mathrm{CTR}}(0.55-1.00 ; 0.14)$ \\
\hline Sys. Pressure & $109(82-132 ; 12)$ & $139(109-178 ; 24)$ & $124(105-145 ; 14)$ & $160^{\mathrm{CTR}, P, H}(118-249 ; 36)$ \\
\hline Dia. Pressure & $68(50-90 ; 9)$ & $88(72-103 ; 11)$ & $76(49-92 ; 11)$ & $98^{\mathrm{CTR}, P, H}(70-149 ; 21)$ \\
\hline IGF-1 (IU/I) & $10.9(2.02-38.3 ; 6.4)$ & $7.50^{P+H}(3.28-16.81 ; 3.74)$ & $5,9(2.44-19.96 ; 4.62)$ & $\mathbf{3 , 4}{ }^{C T R, P}(2.28-11.21 ; 2.70)$ \\
\hline
\end{tabular}

$\mathrm{P}_{;} \mathrm{H}_{;} \mathrm{P}_{\mathrm{i}} \mathrm{H}_{;} \mathrm{CTR}$ - statistical significance comparing to group (respectively): $\mathrm{PIH}, \mathrm{Hypo}, \mathrm{PIH}+\mathrm{Hypo}$ and Control $(\mathrm{p}$ value $<0.05$ )

The groups also differed in the values of anthropometric measurements, birth weight, body length, head circumference and chest circumference. These parameters were, of course, significantly lower in hypotrophic neonatal groups with or without gestational-induced hypertension. There was also a tendency to lower values of these parameters in newborns from the PIH group relative to the control group but no statistical significance was found. It may indicate some risks in this group of patients in which pre-natally no intrauterine growth inhibition was observed. Regression analysis showed a strong negative correlation between systolic blood pressure and the concentration of insulin-like growth factor-1 in the hypotrophic neonatal population from pregnancies complicated by pregnancy-induced hypertension (Tab. 1). A similar tendency was observed in the group of eutrophic neonates from pregnancies with $\mathrm{PIH}$, although without statistical significance. Interestingly, there seem to be differences between groups of newborns with pregnancies complicated by pregnancy-induced hypertension and isolated hypotrophy. In the group of isolated hypotrophy we observed possitive correlation between values of pulsatility and resistance indices and concentrations of IGF-1. We also observed surprisingly positive correlation between antropometric parameters and systolic and diastolic maternal pressure, but there was no correlation between the head circumference and body length and the concentration of insulin-like growth factor-1 in cord blood in this group of patients. In the remaining groups, lower concentrations of IGF-1 were observed with the increase of the pulsatility and resistance indices. In the $\mathrm{PIH}$ and $\mathrm{PIH}+$ Hypo groups, higher values of birth weight and body length, head and
Table 2. Correlation between the concentration of insulin-like growth factor- 1 in cord blood and selected parameters in the tested and control groups

\begin{tabular}{|l|l|l|l|l|}
\hline & Control & PIH & Hypo & PIH + HYPO \\
\hline HBD & 0 & 0.28 & 0 & 0.24 \\
\hline B.W [g] & 0 & 0.44 & -0.31 & 0.30 \\
\hline Lenght & -0.21 & 0.39 & 0 & 0.41 \\
\hline Head circ. [cm] & -0.21 & 0.16 & 0 & 0.18 \\
\hline Chest circ. [cm] & 0 & 0.37 & -0.43 & 0.35 \\
\hline Sys. Pressure [mm Hg] & 0 & -0.24 & 0 & $-0.81 \#$ \\
\hline Dia. Pressure [mm Hg] & 0 & -0.20 & 0 & -0.63 \\
\hline Mean UA PI & -0.27 & -0.42 & 0.20 & -0.47 \\
\hline Mean UA RI & -0.24 & -0.32 & 0.27 & -0.64 \\
\hline
\end{tabular}

\# means statistical significance $(p<0.05)$

chest circumference were observed with an increase in IGF-1 concentration (Tab. 2).

\section{DISCUSSION}

Insulin-like growth factors (IGF-1 and IGF-2) are peptides with structural similarity to proinsulin. The biological role of insulin-like growth factor-1 is omni-directional, with both aut-, para- and endocrine regulation. Its presence is already observed in the early stages of embryonic development and is one of the most important factors of growth, differentiation and maturation of tissues, affecting fetal growth processes. It also seems to be an extremely important factor, in addition to vascular endothelial growth factor (VEGF), Progesterone induced blocking factor (PIBF) and insulin-like 
growth factor-2, which determines normal course of maturation, differentiation and invasion of extravillous trophoblast in spiral arteries, which process, if it goes wrong, is considered the main cause of pregnancy-induced hypertension [9]. The studies also demonstrated the regulatory effect of IGF-1 on endothelial function. It regulates and stimulates the migration of endometrial cells, stimulates neovascularization processes. There are also reports of IGF-1 vasodilatation function in in vivo studies [10]. In animal model studies, low concentrations of IGF-1 were found to be associated with higher blood pressure values due to impaired endothelium-dependent vascular relaxation via nitric oxide and increased expression of vasoconstrictor endothelin [11]. In vitro studies on endothelial cells of human umbilical veins showed a strong effect of insulin and IGF-1 on the synthesis of nitric oxide [12]. It is believed that one of the mechanisms of pregnancy-induced hypertension is the disturbed balance between vasodilators and vasoconstrictors. It is also known that insulin resistance is one of the important risk factor for pregnancy-induced hypertension.

In pregnancies complicated by preeclampsia, a lower IGF-1 concentration was observed as compared to pregnancies with normal maternal blood pressure $[13,14]$, and the severity of preeclampsia was inversely correlated with IGF-1 expression in the placenta and was in direct proportion to expression of Insulin-like growth factors binding protein-3 [15].

In the present study, similar results were also observed with lower IGF-1 concentrations in pregnancies complicated by pregnancy-induced hypotension and/or hypotrophy compared to the control group. Only in groups with pregnancy-induced hypertension concentration of IGF-1 was negatively correlated with maternal arterial pressure and resistance and pulsatility indices in umbilical arteries.

The limitation of this study was lower mean gestational age in groups of isolated $\mathrm{PIH}$ and $\mathrm{PIH}$ with hypotrophy compared with the other groups. It resulted from the necessity to terminate such a complicated pregnancy earlier, both due to the mother's condition (preeclampsia, placental abruption) and threatening fetal asphyxia, which was the most common cause.

However, in the statistical analysis, there was no significant relationship between IGF-1 concentration and gestational age. Similar results were published by S. Sifakis et al. [16]. They did not show statistically significant correlations between gestational age, pulsatility index values in the umbilical arteries and IGF-1 concentration.

The other limitation of this study was the lack of information about biochemical profiles and uterine artery flow in the first trimester. Other limitations included the presence of PIH and/or IUGR in previous pregnancies and the usage of acetylsalicylic acid as a prophylaxis of preeclampsia.
Incorrect placental formation leads to its insufficiency, inadequate supply of nutrients to the fetus and, as a result, to fetal growth restriction. These unfavorable intrauterine conditions result in limitations of cell divisions, changes in metabolic and signaling pathways, and even lead to epigenetic changes. This phenomenon was discribed as Barker's hypothesis [17].

An extremely important role in these processes plays reduced insulin secretion, as well as disturbances in the Growth Hormone/IGF-1 axis. The animal model studies have shown a reduction of pancreatic B-cells mass in growth restricted fetuses [18-20]. An animal model presented by Jones et al. in 1984 showed significant endocrine differences between eutrophic and hypotrophic fetuses. In smaller gestational age fetuses (SGA) they observed hypoglycaemia, hypoinsulinaemia, hypocortyzolemia and hypothyreosis, and they were negatively correlated with severity of growth restriction. In the SGA group there was also higher secretion of androstenedione and glucagon [21]. The studies also showed significantly lower insulin-like growth factor-1 secretion and increased insulin-like growth factor- 2 secretion compared to normal growing fetuses [22].

\section{SUMMARY}

Pregnancy-induced hypertension is strongly associated with the risk of IUGR, and insulin-like growth factor-1 seems to be a common denominator in the pathomechanism of these pregnancy complications, both independently and coexisting. An extremely interesting issue is the increased risk of hypotrophy in the normotensive pregnancy of mothers with history of pregnancy-induced hypertension in previous pregnancies. This phenomenon has not been explained yet, but it has been confirmed in a large cohort study on the Swedish population, which may indicate a common molecular mechanism of both complications of pregnancy [23]. Based on this study it seems that in the case of pregnancy-induced hypertension, the concentrations of insulin-like growth factor-1 are lower with the higher values of maternal systolic and diastolic pressures and pulsatility and resistance indices in umbilical arteries. However, in the case of isolated hypotrophy, despite lower cord IGF-1 concentrations, there was no correlation between maternal blood pressure and IGF-1 concentration. Interestingly, during the study we observed that in the hypotrophy group the IGF-1 concentration was discretely higher at higher values of the umbilical artery resistance and pulsatility indices, which may suggest a completely different pathomechanism. Therefore, it is necessary to broaden the research in this field with the comparison of this two serious complications of pregnancy.

\section{REFERENCES}

1. Xiong $\mathrm{Xu}$, Buekens $\mathrm{P}$, Pridjian $\mathrm{G}$, et al. Pregnancy-induced hypertension and perinatal mortality. J Reprod Med. 2007; 52(5): 402-406, indexed in Pubmed: 17583239. 
2. Baschat AA. Neurodevelopment following fetal growth restriction and its relationship with antepartum parameters of placental dysfunction. Ultrasound Obstet Gynecol. 2011;37(5):501-514, doi: 10.1002/uog.9008, indexed in Pubmed: 21520312.

3. Ananth CV, Basso O. Impact of pregnancy-induced hypertension on stillbirth and neonatal mortality. Epidemiology. 2010; 21(1): 118-123, doi: 10.1097/EDE.0b013e3181c297af, indexed in Pubmed: 20010214.

4. Kornacki J, Skrzypczak J. [Preeclampsia--two manifestations of the same disease]. Ginekol Pol. 2008; 79(6): 432-437, indexed in Pubmed: 18652132.

5. Heimrath J, Czekański A, Krawczenko A, et al. [The role of endothelium in the pathogenesis of pregnancy-induced hypertension]. Postepy Hig Med Dosw (Online). 2007; 61: 48-57, indexed in Pubmed: 17369773.

6. Granger JP, Alexander BT, Llinas MT, et al. Pathophysiology of preeclampsia: linking placental ischemia/hypoxia with microvascular dysfunction. Microcirculation. 2002; 9(3): 147-160, doi: 10.1038/sj.mn.7800137, indexed in Pubmed: 12080413.

7. Figueras F, Caradeux J, Crispi F, et al. Diagnosis and surveillance of late-onset fetal growth restriction. Am J Obstet Gynecol. 2018; 218(2S): S790-S802.e1, doi: 10.1016/j.ajog.2017.12.003, indexed in Pubmed: 29422212.

8. Sieroszewski P, Guzowski G. [Prognostic value of the uterine doppler velocimetry at 20-24 gestation weeks for PIH and IUGR development in pregnancy]. Ginekol Pol. 2005; 76(5): 348-357, indexed in Pubmed: 16145853.

9. Milio LA, Hu J, Douglas GC. Binding of insulin-like growth factor I to human trophoblast cells during differentiation in vitro. Placenta. 1994; 15(6): 641-651, doi: 10.1016/s0143-4004(05)80410-2, indexed in Pubmed: 7824449.

10. Bach LA. Endothelial cells and the IGF system. J Mol Endocrinol. 2015; 54(1): R1-13, doi: 10.1530/JME-14-0215, indexed in Pubmed: 25351818.

11. Tivesten A, Bollano $E$, Andersson I, et al. Liver-derived insulin-like growth factor-I is involved in the regulation of blood pressure in mice. Endocrinology. 2002; 143(11):4235-4242, doi: 10.1210/en.2002-220524, indexed in Pubmed: 12399417.

12. Zeng G, Quon MJ. Insulin-stimulated production of nitric oxide is inhibited by wortmannin. Direct measurement in vascular endothelial cells. J Clin Invest. 1996; 98(4): 894-898, doi: 10.1172/JCl118871, indexed in Pubmed: 8770859.

13. Halhali A, Díaz L, Barrera D, et al. Placental calcitriol synthesis and IGF-I levels in normal and preeclamptic pregnancies. J Steroid Biochem
Mol Biol. 2014; 144 Pt A: 44-49, doi: 10.1016/j.jsbmb.2013.12.014, indexed in Pubmed: 24373797.

14. Olmos A, Díaz L, Avila E, et al. Associations between insulin-like growth factor I, vascular endothelial growth factor and its soluble receptor 1 in umbilical serum and endothelial cells obtained from normotensive and preeclamptic pregnancies. Growth Factors. 2013; 31(4): 123-129, doi: 10.3109/08977194.2013.802692, indexed in Pubmed: 23750889.

15. Dubova EA, Pavlov KA, Lyapin VM, et al. Expression of insulin-like growth factors in the placenta in preeclampsia. Bull Exp Biol Med. 2014; 157(1): 103-107, doi: 10.1007/s10517-014-2502-4, indexed in Pubmed: 24915949.

16. Sifakis $S$, Akolekar R, Kappou D, et al. Maternal serum insulin-like growth factor-I at 11-13 weeks in preeclampsia. Prenat Diagn. 2010; 30(11): 1026-1031, doi: 10.1002/pd.2555, indexed in Pubmed: 20865795.

17. Barker DJ. The fetal and infant origins of adult disease. BMJ. 1990; 301(6761): 1111, doi: 10.1136/bmj.301.6761.1111, indexed in Pubmed: 2252919.

18. Styrud J, Eriksson UJ, Grill V, et al. Experimental intrauterine growth retardation in the rat causes a reduction of pancreatic B-cell mass, which persists into adulthood. Biol Neonate. 2005; 88(2): 122-128, doi: 10.1159/000086136, indexed in Pubmed: 15942163.

19. Mohan R, Baumann D, Alejandro EUy. Fetal undernutrition, placental insufficiency, and pancreatic $\beta$-cell development programming in utero. Am J Physiol Regul Integr Comp Physiol. 2018; 315(5): R867-R878, doi: 10.1152/ajpregu.00072.2018, indexed in Pubmed: 30110175.

20. Bertin E, Gangnerau MN, Bellon G, et al. Development of beta-cell mass in fetuses of rats deprived of protein and/or energy in last trimester of pregnancy. Am J Physiol Regul Integr Comp Physiol. 2002; 283(3): R623-R630, doi: 10.1152/ajpregu.00037.2002, indexed in Pubmed: 12184996.

21. Jones CT, Lafeber HN, Roebuck MM. Studies on the growth of the fetal guinea pig. Changes in plasma hormone concentration during normal and abnormal growth. J Dev Physiol. 1984; 6(6): 461-472, indexed in Pubmed: 6098602.

22. Jones CT. 55Lafeber HN, 55Rolph TP, 55Parer JT. Studies on the growth of the fetalguineapig. The effects of nutritional manipulation on prenatal growth and plasma somatomedin activity and insulin-like growth factor concentrations. J DevPhysiol. 1990; 13(4): 189-197.

23. Wikström AK, Stephansson O, Cnattingius S. Previous preeclampsia and risks of adverse outcomes in subsequent nonpreeclamptic pregnancies. Am J Obstet Gynecol. 2011; 204(2): 148.e1-148.e6, doi: 10.1016/j. ajog.2010.09.003, indexed in Pubmed: 21055722. 